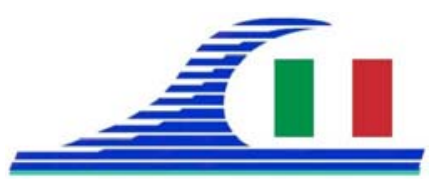

Conférence Méditerranéenne Côtière et Maritime EDITION 3, FERRARA, ITALIA (2015)

Coastal and Maritime Mediterranean Conference

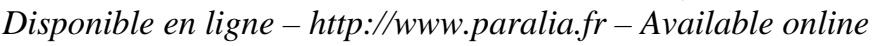

\title{
Etude de la pollution des vases marines des ports algériens par les hydrocarbures
}

\section{Djamila BENYEROU ${ }^{1}$, Nasr Eddine BOUDJENANE ${ }^{1}$, Mansour BELHADRI ${ }^{1}$}

1. Laboratoire de Rhéologie, Transport et Traitement des Fluides Complexes (LRTTFC), Faculté d'Architecture et de Génie civil, Département d'Hydraulique, Université des Sciences et de la Technologie d'Oran Mohamed Boudiaf (USTO-MB) B.P. 1505 Oran-EL-M’naour 31000, Algérie. Benyerou31amri@gmail.com

\section{Résumé :}

Les caractéristiques principales des écosystèmes marins de la Méditerranée diffèrent fortement de celles d'autres mers. Ces caractéristiques déterminent les paramètres chimiques et les paramètres de pollution qui affectent tous les aspects des processus écologiques. Le transport maritime surtout le transport des hydrocarbures favorise l'intervention des risques de pollution pétrolière. La mer Méditerranée est une route importante pour le transport maritime régional et international. Les accidents du trafic maritime et les rejets pétroliers sont les principales causes de cette pollution pétrolière. Cette étude présente les paramètres physico-chimiques et les paramètres de pollution présents dans les sédiments marins du port de Bethioua (Oranie) ainsi que la comparaison entre les ports algériens pollués par les hydrocarbures.

Mots-clés : Vases marines, Port, Caractérisation, Pollution, Dragage, Hydrocarbures.

\section{Introduction}

Les pratiques de dragage constituent un enjeu pour le développement et le maintien des activités portuaires, (BENYEROU et al., 2014). La pollution de ces sédiments par les hydrocarbures est spectaculaire et son impact social, économique et environnemental est lourd, immédiat et à long terme (BOUSQUET, 2003). Les ports qui sont des milieux fermés, favorisent la sédimentation des sédiments et en particulier la fraction fine qui constitue un piège pour les métaux lourds, (BELKESSA et al., 2011). Les caractéristiques principales des écosystèmes marins de la Méditerranée déterminent le devenir des cycles physico-chimiques et biologiques de cette mer. Le développement des activités humaines est devenu aujourd'hui une menace pour l'environnement marin qui subit depuis plusieurs décennies des agressions majeures (ALBAKJAJI, 2011). Les activités maritimes sont génératrices de divers types de pollutions, atmosphériques (cheminées de navires) et marines (substances liquides nocives, cargaison contenant des substances toxiques ou rejets de déchets domestiques). La pollution pétrolière est très dangereuse pour le milieu marin, elle perturbe l'équilibre environnemental de la mer, comme la vie des poissons et les autres êtres vivants. (WALD, 1983). 
Côtes méditerranéennes menacées :

Risques et défis dans le contexte du changement climatique

\section{Matériels et méthodes}

Les sédiments étudiés ont été prélevés au niveau du port de Bethioua (figure 1) qui se consacre aujourd'hui exclusivement aux activités pétrolières. Les sédiments ont été prélevés à l'aide d'une drague mécanique qui est une pelle mécanique montée sur un ponton dans la zone de "Bethioua". Cette zone est classée comme zone pétrolière, où de fortes teneurs en hydrocarbures dans les sédiments ont été constatées. Le matériau dragué a été conservé dans des sachets étanches propres et transféré le jour même du dragage vers les différents laboratoires. L'étude comporte un ensemble de propriétés physiques (masse volumique), de propriétés chimiques ( $\mathrm{pH}$, conductivité, teneur en matière organique, en métaux lourds et en hydrocarbures).

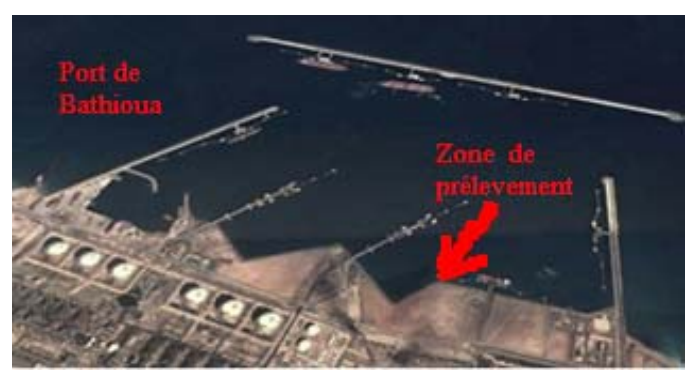

Figure 1. Site de prélèvement des sédiments (Port de Bethioua, Algérie).

\section{Résultats et discussion}

\subsection{Propriétés physico-chimiques}

Le tableau 1 rassemble les propriétés physico-chimiques telles que la masse volumique absolue, le $\mathrm{pH}$, la conductivité et la matière organique. La masse volumique a été obtenue selon la norme NF P94-054, le pH a été déterminé selon la norme NF X31-103, la conductivité électrique a été mesurée avec une cellule conductimétrique étalonnée selon la norme NF ISO 11625 et la mesure de la matière organique a été effectuée en utilisant la norme XP P94-047.

Les éléments traces ont été déterminés par spectrométrie d'absorption atomique. Les résultats obtenus sont rassemblés dans le tableau 2. Ils montrent bien qu’il y a une faible présence des hydrocarbures dans les sédiments marins. Entre les niveaux N1 et N2 des recommandations GEODE (METL \& MATE, 2000), une investigation complémentaire peut s'avérer nécessaire en fonction du projet considéré et du degré de dépassement du niveau N1. Des tests sont pratiqués pour évaluer la toxicité globale des sédiments.

Pour avoir une idée plus précise sur la composition chimique des matériaux étudiés, on a procédé par la fluorescence des rayons $\mathrm{X}$. Les résultats sont regroupés dans le tableau 3 , et exprimés en pourcentage massique. Cette analyse détaillée des résultats est basée sur la comparaison des teneurs pour différents oxydes. 
Tableau 1. Paramètres physico-chimiques des vases marines du port de Bethioua.

\begin{tabular}{ll}
\hline Paramètres & Valeurs mesurées \\
\hline Masse volumique $\left(\mathrm{g} / \mathrm{cm}^{3}\right)$ & 2.2 \\
Matières organiques $(\%)$ & 1.88 \\
$p H$ & 8.7 \\
Conductivité & $361 \mathrm{~ms}$ \\
\hline
\end{tabular}

Tableau 2. Niveaux relatifs aux éléments traces (en $\mathrm{mg} / \mathrm{kg}$ de sédiment sec analysé sur la fraction inférieure à $2 \mathrm{~mm}$ ), METL \& MATE (2000).

\begin{tabular}{lllll}
\hline Métaux & Unités & Valeurs mesurées & Niveau 2 & Niveau 1 \\
\hline Plomb $(\mathrm{Pb})$ & $\mathrm{mg} / \mathrm{kg}$ & $<0.01$ & 200 & 100 \\
Mercure $(\mathrm{Hg})$ & $\mathrm{mg} / \mathrm{kg}$ & $<0.01$ & 0.8 & 0.4 \\
Chrome $(\mathrm{Cr})$ & $\mathrm{mg} / \mathrm{kg}$ & $<0.005$ & 180 & 90 \\
Cadmium $(\mathrm{Cd})$ & $\mathrm{mg} / \mathrm{kg}$ & $<0.01$ & 2.4 & 1.2 \\
Arsenic $(\mathrm{As})$ & $\mathrm{mg} / \mathrm{kg}$ & $<0.05$ & 50 & 25 \\
Etain & $\mathrm{mg} / \mathrm{kg}$ & $<0.01$ & $/$ & $/$ \\
Nitrite & $\mathrm{mg} / \mathrm{kg}$ & 0.00 & 74 & 37 \\
Phénol & $\mathrm{mg} / \mathrm{kg}$ & 0.017 & $/$ & $/$ \\
Hydrocarbures totaux & $\mathrm{mg} / \mathrm{kg}$ & 1.010 & $/$ & $/$ \\
\hline
\end{tabular}

Tableau 3. Composition chimique en éléments majeurs dans les sédiments étudiés.

\begin{tabular}{lllllllllllll}
\hline Paramètre & $\mathbf{S}_{\boldsymbol{i}} \boldsymbol{O}_{2}$ & $\mathbf{A l}_{2} \boldsymbol{O}_{3}$ & $\mathbf{F e}_{2} \boldsymbol{O}_{3}$ & $\mathbf{C a O}$ & $\mathbf{M g O}$ & $\mathbf{S O}_{3}$ & $\boldsymbol{K}_{2} \boldsymbol{O}$ & $\mathbf{N a}_{2} \mathbf{O}$ & $\mathbf{C l}$ & $\boldsymbol{P}_{2} \boldsymbol{O}_{5}$ & $\mathbf{T}_{i} \boldsymbol{O}_{2}$ & $\mathbf{P A F}$ \\
\hline Teneur (\%) & 27.9 & 6.1 & 4.01 & 29.4 & 2.71 & 0.85 & 0.70 & 0.34 & 0.02 & 0.11 & 0.18 & 28.9 \\
\hline
\end{tabular}

3.2 Pollution par les hydrocarbures dans les sédiments des ports algériens

Une pollution par les hydrocarbures est imputable aux rejets d'eaux usées industrielles et urbaines, de boues toxiques provenant de raffineries de pétrole à Alger, Oran et Skikda et de la production de gaz naturel. En raison de la présence d'activités pétrolières en Algérie, les sédiments portuaires et côtiers présentent de fortes concentrations en hydrocarbures pétroliers totaux (tableau 4).

\section{Conclusion}

Cette étude a montré que la mer Méditerranée est menacée par la pollution due à la densité du trafic maritime. Ce trafic, qui s'accompagne du déversement d'hydrocarbures (pollution opérationnelle et accidentelle) conduit à l'augmentation de la dangerosité pour l'écosystème et la biodiversité dans ce bassin semi-fermé. La caractérisation physico-chimique des sédiments de dragage du port de Bethioua présente un taux faible en hydrocarbures par rapport aux autres ports algériens et une absence de pollution métallique. Les compétences de maîtrise et de gestion de la pollution au niveau national et international doivent prendre les mesures nécessaires pour réduire ce phénomène. L'épuration des eaux usées urbaines et des eaux usées industrielles doit répondre à des normes très strictes avant le rejet dans la mer. 
Côtes méditerranéennes menacées :

Risques et défis dans le contexte du changement climatique

Tableau 4. Concentrations d'hydrocarbures pétroliers totaux (HPT) dans les vases portuaires en Algérie.

\begin{tabular}{ll}
\hline Ports & HPT en $\mathbf{~ m g} / \mathbf{k g}$ \\
\hline Oran & $1500-17000$ \\
Arzew & $930-8600$ \\
Bethioua & $67-940$ \\
Mostaganem & $1600-8800$ \\
Ténès & $680-990$ \\
Alger & $1900-31000$ \\
Bejaia & $140-260$ \\
Jijel & $180-430$ \\
Ancien port de Skikda & $450-2000$ \\
Nouveau port de Skikda & $79-120$ \\
Annaba & $130-6200$ \\
\hline
\end{tabular}

\section{Remerciements :}

Les auteurs expriment leurs sincères remerciements à tous les membres du Laboratoire LRTTFC du département d'Hydraulique de l'USTO-MB et du laboratoire de LAFARGE de la cimenterie de SIG (Algérie).

\section{Références bibliographiques}

ALBAKJAJI M. (2011). La pollution de la mer Méditerranée par les hydrocarbures liée au trafic maritime. Thèse de doctorat à l'université de Paris-Est.

BELKESSA R., BACHOUCHE S., BACHARI HOUMA F. (2011). Ensablement et pollution par les métaux lourds des sédiments du port de Ténès. Conférence Méditerranéenne Côtière et Maritime Edition 2, Tanger, Maroc, pp 299-304. http://dx.doi.org/10.5150/cmcm.2011.062

BENYEROU D., BOUDJENANE N., BELHADRI M. (2014). Etude comparative sur les caractéristiques physico-chimiques des sédiments de dragage portuaire et de la barbotine utilisée dans la fabrication de brique. Conférence Matériaux 2014 - Colloque Ecomatériau, Montpellier, France.

BOUSQUET J. (2003). Les risques de pollution par hydrocarbure en méditerranée. Conseil Economique Et Social Régional Languedoc-Roussillon. Montpellier.

METL, MATE - Ministère de l'équipement, des transports et du logement, Ministère de l'aménagement du territoire et de l'environnement- (2000). Arrêté du 14 juin 2000 relatif aux niveaux de référence à prendre en compte lors d'une analyse de sédiments marins ou estuariens présents en milieu naturel ou portuaire. Journal Officiel $n^{\circ} 184 \mathrm{du}$ 10 août 2000, texte $\mathrm{n}^{\circ} 24$, pp 12415-12416.

WALD L. (1983). La pollution pétrolière en Méditerranée vue par le satellite LANDSAT. Méditerranée télédétection III. Ecole nationale supérieure des mines de Paris. 